\section{LUCES Y SOMBRAS DE LA REGENERACIÓN URBANA: PERSPECTIVAS CRUZADAS DESDE LATINOAMÉRICA Y EUROPA}

Mónica Bustos-Peñafiel ${ }^{1}$ y

María Castrillo-Romón²

El número 100 de Revista INVI proporciona una excelente ocasión para debatir sobre la llamada "regeneración urbana" desde una perspectiva cruzada, europea y latinoamericana. Este concepto ha ido ganando una enorme presencia en las políticas urbanas de las últimas décadas a ambos lados del Atlántico, al tiempo que ha ido extendiendo su significado hasta identificarse prácticamente con cualquier tipo de intervención sobre áreas urbanas existentes, sin importar las características urbanísticas de los ámbitos afectados ni la escala, objetivos y herramientas de la acción pública.

1 Instituto de la Vivienda Facultad de Arquitectura y Urbanismo Universidad de Chile, https://orcid.org/0000-00030608-5132.Chile.Correo electrónico: monica.bustos@ uchilefau.cl

\section{LIGHTS AND SHADOWS OF URBAN REGENERATION: CROSSED PERSPECTIVES FROM LATIN AMERICA AND EUROPE}

\author{
Mónica Bustos-Peñafiel y \\ María Castrillo-Romón
}

\begin{abstract}
Esta indefinición creciente no obsta, sin embargo, para que la "regeneración urbana" se presente (y se acepte) con frecuencia como una fórmula virtuosa que, aplicada sobre tejidos edificados, sería capaz de resolver (o eso se pretende) no sólo problemas propiamente urbanísticos (esto es, relacionados con la ordenación del espacio físico) sino también problemas urbanos de naturaleza social o socioeconómica, como son la desigualdad, la exclusión social, el declive económico y muchos otros que se han ido añadiendo a medida que se perfilaban nuevos y cada vez más

\footnotetext{
2 Instituto Universitario de Urbanística (IUU) de la Universidad de Valladolid, https://orcid.org/0000-0002-2331-2854.
} España. Correo electrónico: mariacr@arq.uva.es
\end{abstract}


complejos "retos" para las urbes actuales, como el cambio climático, la migración, el cambio demográfico, etc. (Organisation for Economic Cooperation and Development [OECD], 2013). La "regeneración urbana" se perfila hoy como una panacea para tal diversidad de desafíos que cabe imaginar que, tanto los actuales contextos de crisis social que enfrentan diversos países como las consecuencias aún incalculables que está dejando tras de sí la pandemia también darán lugar a nuevos argumentos y contenidos para su aplicación.

Aunque es dudoso el interés de seguir compilando definiciones en torno a la "regeneración urbana" o de continuar diseccionando matices o diferencias con otros sustantivos de la urbanística contemporánea que también empiezan por re-, creemos que sí vale la pena seguir profundizando en la reflexión sobre el significado y uso de este término.

Es por ello que, a partir de las diversas e interesantes perspectivas que los artículos aquí recogidos abordan, nos ha parecido oportuno discutir la ambigüedad de la "regeneración urbana" y situarla en el contexto histórico en el que cobra sentido para, a partir de ahí, aproximarnos al o los papeles que la "regeneración urbana" está asumiendo en las políticas de desarrollo urbano actuales, identificar lo que su esplendor está dejando en la sombra y, en definitiva, valorar otras tradiciones urbanísticas (principalmente latinoamericanas y de significación mucho más precisa), que tienden a quedar obscurecidas o subsumidas bajo el brillante manto de ese ubicuo término.

\section{La útil pero problemática ambigüedad de un concepto de múltiples significados}

La "regeneración urbana" ha conseguido un lugar preminente en el lenguaje de las políticas públicas urbanas y habitacionales a pesar de ser (o, mejor dicho, por ser) un término enormemente ambiguo, irreductible a una definición única y precisa ${ }^{3}$.

Si miramos de cerca sus múltiples significados, es posible agruparlos al menos en dos, en correspondencia con la doble ontología (la doble relación entre el lenguaje y el ser) que gobierna la cultura occidental, tal como señala el filósofo Giorgio Agamben (2011), y que tensiona las relaciones entre ciencia y política (Sevilla, 2011).

La ontología en indicativo, propia del lenguaje del conocimiento (científico o filosófico), es descriptiva, esto es, re-presenta la realidad (con proposiciones verdaderas o falsas). En el

\footnotetext{
3 Cabe aplicar a la "regeneración urbana" el mismo calificativo que Sylvaine Le Garrec (2006) asignaba al "renouvellement urbain": se trata de nociones "fourretout", a las que se van adhiriendo tantos significados que, finalmente, valen para todo y, por la misma razón, también tienden a quedar vacías de sentido.
} 
caso de la "regeneración urbana", este enfoque subyace a los análisis que intentan dar cuenta objetiva de sus discursos y prácticas, como esta minimalista definición que proponen Isabela Velázquez y Carlos Verdaguer (2011, p. 16).

"Rasgos comunes inherentes al ámbito de la regeneración urbana [...] todas ellas actúan sobre suelo reciclado o que en todas ellas tienen importancia en mayor o menor medida la convergencia entre los sectores público, privado y tercer sector. Igualmente, los mecanismos de participación ciudadana y los criterios de sostenibilidad aparecen bajo una u otra forma".

La ontología en imperativo es, por su parte, prescriptiva y característica del lenguaje legal, religioso y mágico. No corresponde a lo que existe ("es") sino a una voluntad, a una suerte de mandato ("sea"). No es difícil reconocer esta ontología en los poderosos y retóricos discursos institucionales, cada vez más consensuales, donde la "regeneración urbana" aparece como una loable herramienta, y también en la literatura técnica que los parafrasea, donde su definición aparece impregnada, como no puede ser de otro modo, de la ideología de cada autor o cada fuente. Como ilustración, valga esta ambiciosa definición que acompaña a la carta de Toledo:

"La regeneración urbana integrada se concibe como un proceso planificado que ha de trascender los ámbitos y enfoques parciales hasta ahora habituales para abordar la ciudad como totalidad funcional y sus partes como componentes del organismo urbano, con el objetivo de desarrollar plenamente y de equilibrar la complejidad y diversidad de las estructuras sociales, productivas y urbanas, impulsando al mismo tiempo una mayor ecoeficiencia ambiental.

Este concepto de 'regeneración urbana integrada' pretende optimizar, preservar o revalorizar todo el capital urbano existente (social, urbanístico, patrimonio edificado, etc.), en contraste con otras formas de intervención, en las cuales, dentro de este capital urbano, sólo se prioriza y conserva el valor del suelo, al demolerse traumáticamente y sustituirse el resto del capital urbano y, muy lamentablemente, el capital social." (Unión Europea, 2010).

Desde la perspectiva descriptiva de la investigación (perspectiva del conocimiento de la realidad de las cosas, "desde abajo"), el cruce e interacción permanente de estas dos ontologías y la multiplicidad de significados de la "regeneración urbana" constituyen un desafío para el rigor del análisis científico, y obliga a quienes estudian sus problemáticas a un ejercicio casi permanente de delimitaciones semánticas.

Sin embargo, desde la perspectiva prescriptiva de las políticas públicas (una perspectiva "desde arriba", desde el gobierno de las cosas), la maleabilidad del significado de la "regeneración urbana" no solo no es problemática, sino que además resulta muy útil:

"Due to its nature and practice, urban regeneration is far from being a completely fixed set of guiding principles and practices, and does not have a proven or well- established 
track record of success. However, Turok (2005) argued that some ambiguity about the scope and purpose of urban regeneration can be helpful in terms of flexibility and modification. Urban regeneration strategies can be aware of the difficulties arising from 'one size fits all' approaches and of complicated local scenarios and geographies" (Tallon, 2013, p. 6).

Es más, quizá el rasgo más importante y original de la "regeneración urbana" (y la fuente de su éxito internacional en las políticas públicas) sea precisamente su funcional ambigüedad:

"El concepto de regeneración evita una definición precisa y deja un espacio amplio para las ambigüedades y diversas interpretaciones. La regeneración señala normalmente un proceso de renovación, es decir, una cierta forma de reparación o mejora. En el contexto de las políticas públicas, el término se utiliza para describir líneas de acción para transformar un cierto sistema de variables físicas y socioeconómicas" (European Commission, 2006).

\section{Origen y sentido de la regeneración urbana: ¿reducir o reproducir las desigualdades sociales?}

La "regeneración urbana" surge y se desarrolla en un contexto que, desde mediados de los años 1970, ha estado marcado por un modelo de acumulación capitalista nutrido ideológicamente por el neoliberalismo y apoyado en cuatro procesos fundamentales: tecnologización, transnacionalización, flexibilización y financiarización. A menudo es denominado "capitalismo global", si bien la crítica más acerada lo califica como "capitalismo globalitario” (Busquet y Garnier, 2011, p. 45).

Haciendo abstracción de muchas particularidades nacionales y locales, el desarrollo de ese modelo en las ciudades de finales del siglo XX y principios del XXI puede relacionarse con algunos fenómenos urbanos muy importantes y generalizados. En Europa $y$, en menor medida, en Latinoamérica, la llamada "desindustrialización" -esto es, la reestructuración productiva a nivel mundial, con sus consecuencias sobre el volumen y tecnología del transporte global- sentenció a la obsolescencia, al declive o al abandono a innumerables espacios industriales o infraestructurales que, en ocasiones, han sido objeto de grandes operaciones de transformación, denominadas de "regeneración urbana". Isle of Dogs (Londres), Paris-Rive Gauche, Hamburg Hafencity, Villa Olímpica de Barcelona o Bilbao Ría 2000 forman parte de la pléyade de casos más tempranos o conocidos. Lo común a todos ellos es la ubicación relativamente central dentro de sus áreas urbanas de referencia, la formación de partenariados público-privados ${ }^{4}$ para su

$4 \quad$ El anglicismo "partenariado" es un concepto cada vez más extendido para referirse a la asociación público-privada, especialmente en materia de desarrollo urbano. Aunque, desde el ámbito institucional es fuertemente promovido, para la sociología más crítica deja en "evidencia la relación 
desarrollo y el cambio radical de usos del suelo: donde antes había industrias, almacenes o infraestructuras surgen otros usos susceptibles de generar una mayor renta, como actividades económicas terciarias, equipamientos culturales de prestigio y viviendas para clases medias.

Sin embargo, en la otra cara de la moneda, las ciudades europeas también conocieron el agravamiento sostenido de las desigualdades, la pobreza y la exclusión social urbana producidas por el nuevo modelo económico, incluso en los periodos de crecimiento. Así, al tiempo que aquellas nuevas y esplendorosas áreas "regeneradas" emergían con fuerza en la escena urbanística internacional, en las ciudades se acrecentaba la segregación socio-espacial y muchos barrios de las periferias urbanas se iban hundiendo en una profunda crisis. Calificados como "difíciles", "vulnerables", "sensibles", "en crisis", etc., algunos de ellos alcanzan cotas de relegación social con las que escalan puestos en un lamentable ranking internacional que nos negamos a reproducir aquí. Convertidos en un "problema público" (Cefaï, 1996), estos barrios también serán objeto preferente de intervenciones de "regeneración urbana" que, en este caso, no perseguirán cambiar el uso residencial sino que influir en otras dimensiones como el perfil socioeconómico de la población, las tensiones sociales, los conatos de violencia urbana...

de poder que se establece entre los poderes públicos y las potencias privadas, reduciendo al mínimo el papel de las clases populares" (Garnier, 2015).
En las ciudades de Latinoamérica, la dualidad de procesos espaciales y sociales se ha dado con características propias. Con la entrada en la fase del capitalismo neoliberal y globalizado, la región comenzó a acelerar las exportaciones y a conocer una nueva fase de crecimiento económico. Desde los años 1980s, se implementan en diversos países grandes operaciones urbanas de prestigio desarrolladas por la vía de las concesiones y el fortalecimiento de las asociaciones público-privadas ("partenariado"). Esas nuevas lógicas de actuación se vinculan generalmente a costosos proyectos urbanos pilotados por promotores privados para la formación de nuevos centros financieros, comerciales o residenciales de "alto estándar" en áreas en desuso, obsoletas, deterioradas o periféricas que disfrutaban de una localización interesante dentro de la ciudad, potenciada en muchos casos por la construcción de nuevas infraestructuras públicas como autopistas, red de metro, parques, etc. Este es el caso, por ejemplo, de Puerto Madero en Buenos Aires (sobre los terrenos de antiguas dársenas), Lomas de Santa Fe o Nuevo Polanco en Ciudad de México (la primera, construida en parte sobre rellenos sanitarios; la segunda, sobre un área industrial y obrera) o Nueva las Condes en Santiago de Chile (remplazando un conjunto de viviendas sociales de los años 70). En otros casos, las operaciones que buscan propulsar actividades económicas más rentables en "áreas en declive" han puesto su foco sobre los centros históricos 
("revitalización"): Cartagena de Indias, Quito, La Habana, Ciudad de México, Valparaíso, etc.

En el extremo opuesto del espectro social, los barrios informales latinoamericanos siguen creciendo ${ }^{5}$ y, desde los años 1980, comienzan a compartir en el paisaje de las periferias urbanas con extensos y precarios conjuntos de vivienda social formal que proliferan sobre la base de una producción subsidiada por el Estado, orientada a la tenencia en propiedad (México, Colombia, Chile...). Se trata de barrios muy homogéneos y segregados del resto de la ciudad y que, sin mayor dotación urbana, terminan por configurar rápidamente un paisaje de deterioro.

El "mejoramiento" barrial reivindicado por las comunidades de los asentamientos informales se va convirtiendo en una constante de la gestión urbana desde los años 1970 y comienza a institucionalizarse hacia la década de 1990 para, posteriormente, a partir del nuevo siglo, inspirar los mecanismos de participación popular de ciertas políticas públicas de "recuperación" de conjuntos de vivienda social en Chile y México. El caso de Medellín, reconocido en las últimas décadas en la escena internacional de las políticas públicas urbanas, ilustra una inflexión reciente: promover una "recuperación" de las periferias populares basada en grandes proyectos, aunque con un cariz social.

5 Según datos de CEPAL (2018), en promedio, en 2017, el 21\% de la población urbana de América Latina y el Caribe vive en tugurios (United Nations, 2019).
En definitiva, aunque la "regeneración urbana" se haya presentado en ocasiones como una innovación frente a las denostadas políticas públicas de renovación urbana de tabla rasa de las décadas de postguerra (Roberts, 2017; Álvarez Mora y Camerin, 2019), en la actualidad goza de un prestigio que, desde un punto de vista social, es inmerecido, ya que, desde su misma génesis, se viene perfilando como un medio para un desarrollo urbano bipolar y profundamente desigual: por un lado, propulsa nuevas centralidades urbanas y, por otro, se limita a contener el declive o las deficiencias de los barrios más desprotegidos.

\section{Ciudades competitivas y segregación socioespacial: una perspectiva internacional de la "regeneración urbana" de "barrios vulnerables"}

\begin{abstract}
Si bien la transformación del espacio construido es una constante histórica en nuestras ciudades, las operaciones sobre áreas urbanas, promovidas desde la acción pública y en las que la demolición de edificios no es un punto de partida constituyen una novedad histórica propia del siglo XX (Castrillo Romón, 2013). Aunque esta modalidad de intervención empezó a ser aplicada de forma generalizada
\end{abstract}


por Estados europeos y latinoamericanos en sus políticas para barrios populares desde aproximadamente la década de 1970, fue a partir de la década de 1990 cuando se registró otra importante inflexión histórica: grandes agentes internacionales comenzaron a promover la institucionalización de la categoría de "barrios vulnerables" asociada a la "regeneración urbana" o "regeneración urbana integrada".

La Unión Europea, fundada en 1991 como institución pública supranacional que se daba como "imperativos políticos" el desarrollo sostenible y la cohesión social, reconocía sin ambages la brecha social que abría el crecimiento económico:

"La actual aparición de nuevas oportunidades económicas está contribuyendo en muchas ciudades a agrandar las disparidades sociales y económicas. [...] La situación de penuria de las ciudades se refleja en el aumento de la pobreza, el incremento de las personas sin techo, el aislamiento social, las infimas condiciones de alojamiento, el abuso de estupefacientes y las conductas delictivas.

En muchas ciudades europeas, el fenómeno de exclusión ha conducido a la segregación física de determinados grupos sociales a los barrios peor equipados. [...] Además, en muchas ciudades la exclusión social se superpone a la diversidad cultural y lingüística de muchos barrios en los que el sistema educativo presenta requisitos especiales." (Comisión de las Comunidades Europeas, 1997, p. 197) [énfasis agregado].
Para hacer frente a esta situación, la Comisión Europea impulsó, desde 1994 hasta 2006, la iniciativa comunitaria URBAN (I y II). Su finalidad era financiar programas de "desarrollo integrado" para áreas urbanas concretas identificadas como "zonas desfavorecidas" ("Comunicación de los estados miembros", 1994) y, a través de ello, promover en Europa un modelo de intervención (la "regeneración urbana integrada") que arraigaba en una noción de "regeneración urbana" forjada inicialmente en el Reino Unido (Gregorio, 2012).

Es importante observar que la Comisión Europea no buscaba actuar sobre el origen de las desigualdades sociales o urbanas, sino promover un modelo de intervención que atenuara las situaciones más deplorables y evitar así que influyan negativamente sobre la capacidad de la ciudad para captar inversiones:

"Cada vez se encuentra más extendida la opinión de que la segregación territorial no sólo constituye un problema en términos de empleo, educación y mala calidad de la vivienda, sino que, además, los comportamientos asociales que de ella se derivan resultan perjudiciales para el interés y el atractivo económico de la ciudad" (Comisión de las Comunidades Europeas, 1997, p. 197)

"La Comisión aboga por dar un enfoque zonal a la regeneración de áreas urbanas deprimidas dentro de los Fondos estructurales, 
integrando aspectos económicos, sociales, culturales, medioambientales, de transporte y de seguridad. Igualmente, importante es relacionar las áreas urbanas con dificultades con las estrategias sociales y económicas más generales a fin de evitar la segregación urbana" (Comisión Europea, 1998) [énfasis agregado].

En Latinoamérica, aunque la erradicación de los barrios informales fue el horizonte nominal de las políticas públicas desde la década de 1950, el mejoramiento barrial ha sido, a pesar de su escasa institucionalización, el fenómeno más masivo e importante para las periferias populares desde los años 1970. No será hasta fines del siglo XX que las políticas públicas se involucrarán formalmente en ese tipo de procesos en los que algunos verán un "nuevo" paradigma ${ }^{6}$. Nacerán así el "Programa comunitario de mejoramiento barrial" en México, el "Programa de mejoramiento de barrios" en Chile, el "Programa favela bairro" en Brasil (Río de Janeiro), el "Programa de mejoramiento integral de barrios" de Colombia o el "Programa de mejoramiento de barrios" de Uruguay, entre otros, financiados con recursos públicos (centrales, estaduales o locales) y complementados en muchos casos

6 "Aunque en sus orígenes la política pública de muchos de estos países consideraba que los asentamientos precarios debían ser sustituidos por proyectos residenciales nuevos en las periferias urbanas (Brakarz, Greene y Rojas, 2002), desde la década de los años 90 y principios de la década del 2000 se genera un cambio de paradigma a partir del cual surgen los programas de mejoramiento de barrios (Rojas, 2009, p. 1). con préstamos del Banco Interamericano de Desarrollo (BID) en la región.

De hecho, en Latinoamérica, la influencia del BID como institución supranacional (en este caso, de carácter privado), ha sido crucial desde la década de 1990, principalmente en la difusión de determinados modelos de intervención inspirados en el mejoramiento de barrios. Es interesante observar que la interpretación del problema al que se enfrenta es realmente muy próxima a la que hacía la UE al otro lado del Atlántico:

"Entre los desafíos destacan: las persistentes dificultades de inserción en la economía de las ciudades que enfrentan los habitantes de asentamientos informales; el aumento de los comportamientos antisociales y violentos, que afectan con mayor frecuencia a los asentamientos informales, y el deterioro físico $y$ de las relaciones sociales que se observa en barrios y edificios de departamentos de viviendas sociales construidos por los gobiernos, producto de la marginación física, económica y social de sus ocupantes" (Rojas, 2009, p. xiii) [énfasis agregado]..

"La ciudad informal se entrelaza con todas las dimensiones de la ciudad formal, afectando los mercados laborales, de bienes y servicios, y las relaciones sociales y el territorio. Los problemas de los asentamientos informales afectan tanto a los habitantes de los propios asentamientos como a los de barrios vecinos y a toda la ciudad" (Rojas, 2009, p. 12) [énfasis agregado] 
Bajo la idea de que "sólo una parte de los habitantes de los asentamientos informales de una ciudad han tenido la oportunidad de convertirse en ciudadanos completos", el BID plantea "considerar problemas que hasta ahora han permanecido fuera o en la periferia de la preocupación de los promotores y ejecutores de los programas de mejoramiento de barrios" como, entre otros, impedir la proliferación de barrios irregulares y facilitar y ampliar la incorporación de los beneficiarios a la economía de la ciudad; en definitiva, "ampliar el alcance y la escala de las intervenciones en mejoramiento de barrios para proveer los beneficios de la ciudadanía a todos los habitantes irregulares y para impedir que otros la pierdan gradualmente" (Rojas, 2009, p. 2).

En esa línea, algunos Estados latinoamericanos, como Brasil y Chile, han comenzado a producir una reflexión e indagación en el marco de la modernización del Estado (Jordán y Simioni, 2003), y a instalar lógicas de focalización territorial para intervenir en el mejoramiento de vecindarios $y$ barrios denominados como "vulnerables" o "críticos", etc. bajo los postulados de la sostenibilidad y la construcción de ciudadanía.

En este mismo escenario, a partir del nuevo siglo, especialmente en países como Chile y México, los barrios de vivienda formal de baja calidad promovidos por las políticas públicas "cuantitativas" de las últimas décadas del siglo XX también han empezado a constituir, como en Europa, el objeto de otra modalidad de "regeneración" impulsada, en este caso, desde los gobiernos nacionales. Chile destaca en este ámbito por promover sistemáticamente desde 2006 una "política urbano-habitacional de mejoramiento de calidad e integración social" que se ha implementado con dos programas de referencia: "Quiero mi barrio", centrado principalmente en el mejoramiento del espacio público, y el "Programa de protección al patrimonio familiar", que busca atender el deterioro y conflictos sociales que afectan especialmente a bloques de vivienda social en altura, distintivos de las periferias chilenas, y que han dado paso al diseño de otros diversos programas de regeneración.

Estas iniciativas en nombre de la regeneración urbana, asociadas a las "medidas para implementar una política de suelo para la integración social urbana" (Consejo Nacional de Desarrollo Urbano [CNDU], 2015), han venido a instalar como un elemento clave del debate sobre políticas urbanas el "nuevo papel del Estado en la regeneración de la ciudad segregada". Habiendo aceptado de forma explícita que la regulación es insuficiente para revertir la actual desigualdad urbana, la regeneración de la ciudad viene a perfilarse en el

$7 \quad$ El objetivo del programa es "contribuir al mejoramiento de la calidad de vida de los habitantes de barrios que presentan problemas de deterioro urbano, segregación y vulnerabilidad social, a través de un proceso participativo de recuperación de los espacios públicos y de los entonos urbanos de las familias" (Decreto 14, 2017) 
discurso público chileno como "la" herramienta para superar la segregación urbana, apoyada por la inversión en infraestructura y por la gestión pública del suelo (CNDU, 2015).

Estas perspectivas cruzadas entre Europa y Latinoamérica constatan el avance internacional de la institucionalización de la "regeneración urbana" como una herramienta destacada de la intervención pública sobre los barrios más empobrecidos o deteriorados con el objetivo de contribuir a un mejor posicionamiento de las ciudades en un contexto de competencia internacional. Queda por saber, más allá de los discursos, qué capacidad efectiva puede acreditar la "regeneración urbana" frente a la segregación socioespacial de nuestras ciudades y, lo que es más importante, frente a la satisfacción de las necesidades habitacionales y urbanas expresadas por las comunidades que habitan esos barrios denominados "vulnerables".

8 Los países de la Unión Europea no parecen especialmente preocupados por evaluar sus políticas de regeneración urbana integrada: una encuesta realizada en 2010 desvelaba que sólo el 45\% hacía algún tipo de seguimiento o evaluación ("Regeneración urbana integrada", 2010) y solo un tercio incorporaba "agentes sociales" o "representantes de los habitantes afectados" en sus evaluaciones.

\section{Luces y sombras de la "regeneración urbana" en barrios populares. Por una revalorización de la tradición del mejoramiento barrial en Latinoamérica}

La "regeneración urbana" que se ha ido instalando desde las políticas públicas como una modalidad de acción pública llena de ventajas para resolver los problemas de "barrios vulnerables" es, en realidad, un conglomerado de instrumentos y prácticas de intervención lleno de luces y sombras.

En primera instancia, ya desde la focalización de las intervenciones, conviene subrayar que esa "regeneración urbana" se justifica en la mayoría de los casos por las malas condiciones sociales de la población. Esto constituye, por un lado, un desplazamiento (o una superposición) de las políticas urbanas hacia las políticas sociales y, al tiempo, una inversión histórica de la acción social del Estado: legitimado por el aumento sin parangón de la segregación socioespacial urbana, el objetivo tradicional de las políticas sociales, que eran los grupos sociales más débiles, se trasmuta en los barrios donde éstos se concentran en mayor proporción. 
Por otro lado, la identificación de los ámbitos susceptibles de ser "regenerados" suele hacerse por medio de sofisticados mecanismos estadísticos concebidos para que la acción se focalice, en cada ciudad y en cada país, sobre los territorios que presenten los peores indicadores socioeconómicos. Estos procedimientos de clasificación, además de prescindir de la percepción de los propios habitantes de los barrios, suelen contribuir a la estigmatización de los territorios que habitan y, así, a hacerlos víctimas de una doble relegación: por un lado, la relegación urbana que revelan las estadísticas y, por otro lado, la relegación simbólica que supone tanto la negación del valor de su propia perspectiva sobre el barrio como la desvalorización de éste en el imaginario público de la ciudad.

Se ha instalado asimismo el discurso de la participación ciudadana, a través del cual la regeneración urbana pretende actuar. Sin embargo, producto de la institucionalización en la cual han devenido los procesos de participación, ésta ha quedado convertida, en muchos casos, en un mecanismo de legitimación de decisiones previamente tomadas desde "arriba". Incluso en aquellos casos en que la regeneración ha tenido en origen un apoyo popular, ha sucedido que la dimensión financiero-inmobiliaria ha terminado siendo dominante y los intereses de la población han quedado relegados (Cócola, 2016, p. 34-40)
Está también el discurso de la integralidad de la regeneración urbana, referido al objetivo de abordar multidimensionalmente las problemáticas de los barrios y alcanzar la triple sostenibilidad (social, ambiental y económica) de las intervenciones a través de la articulación de actores, sectores, escalas y fuentes de financiación. Pero se trata de un discurso que tampoco está exento de contradicciones. Por un lado, está el hecho de que, aunque la vulnerabilidad socio económica es comúnmente la justificación para la acción pública regeneradora, el mayor esfuerzo de financiación no se suele depositar en las medidas propiamente sociales sino, sobre todo, en las acciones edificatorias ("HighLevel Conference", s.f.). Por otro lado, en muchos casos, los plazos electorales marcan las intervenciones y comprometen la continuidad de esas múltiples articulaciones, con lo que también afectan a las oportunidades de éxito de las acciones planteadas (Matesanz, 2016). Finalmente, aunque la coordinación interadministrativa de la planificación y la gestión urbana integradas forman parte de las intenciones de la regeneración urbana, muchas de las intervenciones se terminan abordando de manera aislada, sin consideraciones interescalares o intersectoriales que logren resolver problemas que vayan más allá de aspectos puntuales dentro de un determinado barrio o territorio. 
Esta ausencia de consideración simultánea de diversas dimensiones en la intervención de regeneración de barrios y territorios vulnerables no sólo da cuenta de la escasa voluntad o capacidad de articular multisectorialmente las actuaciones de alcance público, sino que también afecta directamente en el impacto de las acciones de regeneración y, en ciertos casos, en el rápido retorno (o persistencia) del deterioro. No es raro que los habitantes de barrios "regenerados" perciban que, tras el paso de diversas instituciones, profesionales y recursos, nada cambia realmente en sus condiciones de vida.

En definitiva, la institucionalización consolidada de la mano del propio modelo económico ha llevado a la regeneración urbana a constituirse en una forma de intervención definida y configurada desde "arriba", en el que la idea de "integralidad" como principio no logra cumplirse y cuyos resultados efectivos, especialmente en el plano social, están hoy sometidos a un permanente cuestionamiento, no solo por parte de los movimientos sociales y la investigación más crítica, sino que incluso por parte de algunas importantes instituciones como el Banco Mundial (World Bank, s.f.)

A partir de estas sombras e imprecisiones de la "regeneración urbana", es que la noción del mejoramiento urbano aparece como un tipo de intervención característico del contexto latinoamericano cuya trayectoria y logros vale la pena revisitar y reivindicar. Como una herramienta de intervención en asentamientos precarios, el mejoramiento barrial ha ido siendo implementado por la acción pública desde los años 1970 bajo la presión de las reivindicaciones de los propios habitantes que, guiados por necesidades de la vida cotidiana, han introducido una lógica de intervención comunitaria, multidimensional y "bottomup". Desde este enfoque, aunque en muchos casos las intervenciones terminan por reposar sobre la capacidad de gestión de comunidades que se mueven entre la insuficiencia y la precariedad de recursos, y que tropiezan con muchas dificultades para poder influir más allá de los límites de sus propios barrios cuando los problemas se derivan de escalas superiores, la integralidad avant-la-lettre y la intersectorialidad resultan ser consustanciales al mejoramiento barrial y un valor de este tipo de actuaciones.

Es quizás por esto que la tradición del mejoramiento de barriosinformales hainspirado la "recuperación" de otros tipos de barrios que son resultado de distintas modalidades de acción pública en América Latina (políticas de vivienda convencionales y no convencionales). En este campo, en el que se reúnen muy diversas problemáticas y requerimientos (tipo de política o programa, época de construcción...), parece esbozarse la oportunidad de concebir un tipo de intervención con carácter propio y original. 
Dela mano dela reivindicación del mejoramiento como un tipo de intervención propiamente latinoamericana, nos interesa también dar cuenta cómo el actual contexto de pandemia y crisis social ha comenzado a desvelar otras múltiples expresiones urbanas comunes a la región que sería relevante considerar respecto de intervenciones futuras sobre la ciudad ya construida. Como problemáticas comunes, ha aumentado la evidencia respecto del déficit del alojamiento ${ }^{9}$ (personas viviendo en las calles, tomas de terrenos, aumento de asentamientos irregulares...), el allegamiento y el hacinamiento, el subarriendo (especialmente como consecuencia de la migración), la precariedad y la informalidad en sus múltiples facetas: comercio callejero, ollas comunes, etc. Pero también, como valores propios, ha quedado en evidencia la capacidad de las redes comunitarias para activarse rápidamente y el gran potencial de la organización social. La informalidad se revela con la pandemia y, dentro de lo malo de la situación, deja al descubierto un valor inefable: la capacidad de autoorganización vecinal, el fortalecimiento de las redes sociales y sus posibilidades de proyectarse sobre la mejora y autogestión de los barrios o del hábitat en general.

9 En plena cuarentena, el pasado 28 de mayo de 2020, el ex ministro de Salud de Chile, Jaime Mañalich, reconoció: «Hay un nivel de pobreza y hacinamiento del cual yo no tenía conciencia la magnitud que tenía". ("Mañalich asegura", 2020).
Esta realidad nos ofrece hoy un llamado de atención y una nueva oportunidad para volver a revisitar la tradición del mejoramiento urbano como herramienta de intervención, valorar el conocimiento cimentado a lo largo de décadas por comunidades, profesionales/funcionarios públicos e investigadores comprometidos, y superar las ambigüedades y deficiencias de todo tipo que hoy encontramos en la "regeneración urbana" instalada como modelo y doctrina desde las instituciones. De aquí la importancia de poner atención a quienes "desde abajo" denuncian la dominación que se establece a través del lenguaje del poder, colaborando así en reconquistar la ontología en indicativo que la Academia nunca ha debido descuidar.

\section{Aportes del número 100 de INVI: regeneración(es) urbana(s) y nuevas perspectivas sobre el mejoramiento barrial}

La regeneración urbana es un concepto muy polisémico bajo el que se promueven acciones públicas que no son "neutras ni despolitizadas". Así lo evidencia Víctor Delgadillo en "Regeneración urbana en la Ciudad de México: polisemia de concepciones y de acciones públicas" a través de la revisión de un amplio repertorio de referencias fundamentales desde 
la década de 1970. Delgadillo identifica, dentro de ese ambivalente campo semántico, un hilo conductor entre diversas experiencias de intervención sobre tejidos urbanos (de México a Alemania, pasando por Francia e Italia) que tienen como denominador común dos elementos cruciales no siempre presentes en las actuaciones de regeneración urbana: el enfoque holístico y la participación de los habitantes en todas las fases del proceso sobre los barrios -incluida la toma de decisiones. En ese marco sitúa cuatro experiencias de mejoramiento barrial en Iztapalapa, Ciudad de México (PCMB, 2007-2017) que, pese a sus indudables valores, tropiezan con enormes dificultades para salir adelante: Miravalle, Bellavista, Xalpa y Desarrollo Urbano Quetzálcoatl. Finalmente, Delgadillo nos invita a replantear la cuestión desde un ángulo distinto y bien pertinente: "iregeneración urbana para quiénes y según quiénes?"

Las modalidades de regeneración urbana practicadas en Latinoamérica son el eje de artículo "Regeneración urbana: un panorama latinoamericano", de Catherine Paquette. Este texto se interesa por las diferencias con la regeneración urbana predominante en Europa y Norteamérica, y dibuja un interesante panorama latinoamericano donde los grandes proyectos sobre suelos urbanos reciclados son minoritarios, donde la revitalización de los centro históricos y la densificación de las áreas pericentrales tienen un encaje problemático, y donde el "mejoramiento urbano integral de barrios" en periferias urbanas populares (de origen informal pero también, más recientemente, formal) constituye la modalidad de regeneración más abundante y valiosa, merecedora de ser "fuente de inspiración para la elaboración de propuestas alternativas" frente a la regeneración urbana basada en grandes proyectos.

En muchos casos, detrás de las actuaciones desarrolladas en el marco de la institucionalidad ("desde arriba") se forman nuevas, múltiples y silenciosas disputas en la ciudad que dan origen a la formación y movilización de diversos grupos y organizaciones. En "El rol de la sociedad civil en la gestión de vivienda y barrios vulnerables en Chile", Luz María Vergara observa el creciente número de organizaciones de la sociedad civil (OSC) que, a través de la participación en la implementación de proyectos relacionados con el entorno construido, se constituyen en agentes de transformación política y social; además, define y caracteriza su rol en el campo de la vivienda y la regeneración de barrios vulnerables. El marco analítico que propone está basado en la capacidad de intermediación de estas organizaciones y en el tipo de innovación social a la que apuntan; sus conclusiones valoran la actual contribución de estas organizaciones desde su doble papel de implementadores y catalizadores, con un rol predominante en la dimensión de procesos y empoderamiento. 
La asociación público-privado o "partenariado", y el "empresarialismo urbano" son dos nociones estrechamente asociadas a la interpretación dominante de la regeneración urbana. El artículo de Carolina González, "Gobernanza urbana: reflexiones a partir de los distritos económicos de la ciudad de Buenos Aires" se centra en el accionar de los actores públicos para discutir esas formas de gobernanza aplicadas a operaciones de transformación urbana. Tomando como caso de estudio la implementación de cuatro nuevos distritos económicos bonaerenses y adoptando un abordaje cualitativo, la autora nos muestra, por un lado, que la creación de un "clima de negocios" no solo comporta cambios de uso y beneficios impositivos a favor de los agentes privados, asi como una inversión pública muy grande (sin contrapartida privada equivalente) y desigual en infraestructura, transporte, espacio urbano y equipamiento. También conlleva todo un entramado discursivo en el que los poderes locales aparecen como mediadores o facilitadores, y en el que los relatos dominantes contribuyen a legitimar los cambios impuestos y resemantizar las áreas afectadas. Por otro lado, en un segundo plano de análisis, los resultados obtenidos también invitan a repensar los límites y alcances de la noción de "empresarialismo urbano" fuera de los países del "Norte global".

La diversidad de acepciones de la regeneración urbana se extiende a las diferentes escalas de referencia de las intervenciones. El trabajo
"Regeneração e resiliência: as intervenções urbanas recentes na Praça Roosevelt em São Paulo", de las autoras Marlon Paiva y Maria Cristina da Silva Schicchi, tiene como caso de estudio un conocido espacio público del centro histórico de la capital paulista. Conforme a los dos conceptos priorizados en el título, el análisis describe primero las políticas de recualificación que han afectado a esa plaza en las últimas décadas y discute el carácter de "regeneración urbana" de las intervenciones más recientes. A partir de ahí, hace una aproximación al espacio vivido y a los usos y apropiaciones de la plaza, así como a los conflictos, expulsiones (gentrificación) y adaptaciones derivados de las transformaciones del entorno edificado, identificando las acciones de resiliencia de las comunidades de usuarios de la plaza como un indicador de los efectos del modelo dominante de producción de la ciudad contemporánea.

Dos artículos se acercan al análisis de algunos de los actuales programas de escala barrial implementados en Chile con enfoque de regeneración. En "Regeneración urbana y gestión del riesgo en Chile: análisis comparativo de casos", los autores Juan Pablo Sarmiento, Antonio Fritis y Carmen Paz Castro, proponen una aproximación al programa "Quiero mi barrio" desde la perspectiva de la incorporación de medidas de gestión del riesgo en barrios expuestos a riesgos socionaturales. A partir de un análisis comparativo de casos, el articulo muestra que es posible transversalizar medidas 
de reducción de riesgos en el diseño de obras de infraestructura e intervenciones sociales dentro de las operaciones de regeneración urbana, concluyendo que, en ciertos casos, la experiencia del $\mathrm{PQMB}$ ha contribuido efectiva y eficientemente a la seguridad del barrio y de su comunidad, promoviendo, además, el fortalecimiento de la participación ciudadana y los procesos de gobernanza a niveles nacionales, regionales, locales.

Por su parte, los autores de "Consideraciones para programar la regeneración de condominios sociales en altura. Estudio comparado de tres casos en Chile", Francisco Chateau, Cristian Schmitt, Alejandra Rasse, y Paula Martínez, parten del análisis del modelo de gestión del Programa de Regeneración de Conjuntos Habitacionales del MINVU y de los aprendizajes levantados en sus primeras experiencias, para constatar, por medio de un análisis comparativo, una serie de problemáticas derivadas de los largos plazos de ejecución de estos proyectos y, finalmente, identificar algunas dimensiones en la gestión, diseño e implementación en los procesos que permitirían ajustar sus plazos de aplicación.

La monografía se cierra con el artículo de opinión de Francesc Peremiquel, "Hacia una regeneración urbana 2.0. Barcelona como referencia", donde el autor da cuenta de la regeneración urbana llevada a cabo durante los últimos cuarenta años, poniendo en valor los métodos e instrumentos, que, con nombres distintos, pero con finalidades equivalentes, han sido utilizados en la práctica urbanística para este objetivo. A partir de los estudios realizados en diversas tesis doctorales sobre la experiencia de Barcelona y su entorno, el autor distingue dos periodos que forman parte de una misma manera de hacer y tienen un similar instrumental de actuación: el del regreso a la ciudad basado en la urbanidad como objetivo central y el de la sostenibilidad como principio básico de desarrollo.

Finalmente, como cierre del número 100 de la Revista INVI, saludamos con entusiasmo la traducción al castellano, hecha por Luis Campos Medina y Malena Bastías Sekulovic, de un artículo clásico de la Sociología urbana francesa: "Proximidad espacial y distancia social. Los grandes conjuntos de vivienda social y su población", de Jean-Claude Chamboredon et Madeleine Lemaire, publicado inicialmente en la Revue française de Sociologie (vol. 11, n 1) en 1970. La traducción viene precedida de una "Presentación de "Proximité spatiale et distance sociale » para la revista INVI", realizada por Paul Pasquali, sociólogo del Centre National de la Recherche Scientifique de Francia, que viene desarrollando desde hace años una interesante aproximación a las ciencias sociales contemporáneas desde una perspectiva histórica.

Elaborado en el periodo de auge de lo que podríamos llamar la política de vivienda 
social cuantitativa en Francia, el artículo de Chamboredon y Lemaire analiza la mezcla social que ésta promovía y lo hace en su momento álgido, pocos años antes de que el rechazo y la huida masiva de las clases medias sentenciase el eclipse político del modelo y el declive social generalizado de los barrios de bloques y torres en periferia. Como retrato de ese instante antes de la decadencia, "Proximidad espacial y distancia social..." es una referencia frecuente entre los estudiosos de las problemáticas relacionadas con la "regeneración" (renovación, renouvellement...) de grands ensembles de vivienda social en Francia.

\section{Referencias bibliográficas}

Agamben, G. (2011). What is a commandment? Backdoor Broadcasting Company. Recuperado de https://backdoorbroadcasting.net/2011/03/ giorgio-agamben-what-is-a-commandment/

Álvarez Mora, A. y Camerin, F. (2019). La herencia del urban renewal en los procesos actuales de regeneración urbana: el recorrido renovación-regeneración a debate. Ciudad y territorio. Estudios territoriales, 51(199), 5-26. Recuperado de https://recyt.fecyt.es/index.php/CyTET/ article/view/76712

Brakarz, J., Greene, M., y Rojas, E. (2002). Ciudades para todos. La experiencia reciente en programas de mejoramiento de barrios. Washington DC: Banco Interamericano de Desarrollo.
Busquet, G. y Garnier, J. P. (2011). Un pensamiento urbano todavía contemporáneo. Las vicisitudes de la herencia lefebvriana. Urban, (2), 4157. Recuperado de

http://polired.upm.es/index.php/urban/ article/view/1490/1986

Castrillo Romón, M. (2013). La réhabilitation urbaine: une politique impossible? En C. Vaz, C. Vorms y L. Coudroy de Lille (Eds.), L'urbanisme espagnol depuis les années 1970: la ville, la démocratie et le marché (pp. 113-125). Rennes: Presses universitaires de Rennes..

Cefaï, D. (1996). La construction des problèmes publics. Définitions de situations dans des arènes publiques. Réseaux, 14(75), 43-66. https://doi.org/10.3406/reso.1996.3684

Cócola, A. (2016). La producción de Barcelona como espacio de consumo. Gentrificación, turismo y lucha de clases. En Grupo de Estudios Antropológicos La Corrala (Coords.), Cartografía de la ciudad capitalista. Transformación urbana y conflicto social en el Estado español (pp. 31-56). Madrid: Traficantes de Sueños.

Comisión de las Comunidades Europeas. (1997). Hacia una política urbana para la Unión Europea. COM (97)197 final.

Comisión Europea. (1998). Marco de actuación para el desarrollo urbano sostenible en la Unión Europea. COM (1998) 605 final.

Comunicación de los estados miembros 94/C 180/02. (1994, 1 de julio). Diario Oficial de las Comunidades Europeas (C180/6) 
Consejo Nacional de Desarrollo Urbano. (2015). Propuestas para una política de suelo para la integración social urbana. Informe Final. Recuperado de https://cndu.gob.cl/wp-content/ uploads/2015/05/Documento Final Propuesta-de-Poli\%CC\%81ticas Suelo para Integracio\%CC\%81n Social CNDU Mayo 2015.pdf

Decreto 14. Reglamenta Programa de Recuperación de Barrios, Santiago, Chile, 12 de abril de 2007. Recuperado de

https://www.minvu.cl/elementos-tecnicos/decretos/ ds-n14-v-y-u-de-2007-act-24-05-2017/

European Commission. (2006). State aid control and regeneration of deprived urban areas. Vademecum. Recuperado de https:/lec.europa.eu/competition/state aid/studies reports/vademecum.pdf

Garnier, J. P. (2015). Urbanismo y neoliberalismo en Francia: una regresión interminable. Ciudades, (18), 183-196.

https://doi.org/10.24197/ciudades.18.2015.183-196

Garrec, S. I. (2006). Le renouvellement urbain, la genèse d'une notion fourre-tout. La Défense: Plan Urbanisme construction architecture.

Gregorio, S. d. (2012). Politicas urbanas de la Unión Europea desde la perspectiva de la planificación colaborativa. Las Iniciativas Comunitarias URBAN y URBAN II en España. (Tesis doctoral Universidad Politécnica de Madrid, España). Recuperado de http://oa.upm.es/12740/

High-Level Conference results on "Urban Sustainability and Integrated Urban Regeneration in Europe: Policies, Programs and Best Practices", Madrid, 26-27 April 2010". (s.f.).
Recuperado de https://www.eukn.eu/events/ detail/high-level-conference-results-on-quoturban-sustainability-and-integrated-urban-regeneration-in-eur/

Jordán, R. y Simioni, D. (Comps.). (2003). Gestión urbana para el desarrollo sostenible en América Latina y el Caribe. Santiago de Chile: CEPAL. Recuperado de https://repositorio.cepal.org/ handle/11362/2376

Mañalich asegura que "no tenía conciencia de la magnitud" del hacinamiento en Chile. (2020). The Clinic. Recuperado de https://www.theclinic.cl/2020/05/28/manalich-asegura-que-no-tenia-conciencia-de-la-magnitud-del-hacinamiento-en-chile/

Matesanz, A. (2016). Políticas urbanas y vulnerabilidad. (Tesis doctoral Universidad Politécnica de Madrid, España). Recuperado de http://oa.upm.es/44893/

Organisation for Economic Cooperation and Development. (2013). OECD Urban Policy Reviews, Chile 2013. Paris: OECD Publishing. https://doi.org/10.1787/9789264191808-en.

Regeneración urbana integrada en Europa. Documento de síntesis. (2010). Valladolid: IUU. Recuperado de https://www.mitma.gob.es/ recursos $\mathrm{mfom} / \mathrm{pdf} / \mathrm{CODA} 6769-76 \mathrm{CB}-4 \mathrm{~F} 02$ 8937-F5CAF2CCD016/111525/3 survey on integrated.pdf

Roberts, P. (2017). The evolution, definition and purpose of urban regeneration. En P. Roberts, H. Sykes y R. Granger. Urban regeneration (2a edición, pp 9-43). London: Sage. 
Rojas, E. (Ed.). (2009). Construir ciudades. Mejoramiento de barrios y calidad de vida urbana. Washinton, DC: BID, Fondo de Cultura Económica.

Sevilla, A. (2011). Cenizas de Gotha, espectros de Lefebvre: derecho a la ciudad y urbanismo alternativo en el ocaso del Estado social. Geocrítica, 16(932(3)). Recuperado de http://www.ub.edu/geocrit/b3w-932/b3w-932-3.htm

Tallon, A. (2013). Urban regeneration in the UK (2nd. ed.). London: Routledge.

Unión Europea. (2010). Documento de referencia de Toledo sobre la regeneración urbana integrada y su potencial estratégico para un desarrollo urbano más inteligente, sostenible y socialmente inclusivo en Europa. En Reunión informal de ministros de desarrollo urbano. Declaración. Toledo, 22 de junio de 2010. Recuperado de https://www.mitma.gob.es/recursos mfom/ pdf/8343F582-2844-4F9C-BDEF-4C2E2AFC547C/111530/6 declaracion Toledo.pdf

United Nations. (2019). World urbanization prospects: The 2018 revision. New York: United Nations. (ST/ESA/SER.A/420).

Velázquez, I. y Verdaguer, C. (2011). Regeneración urbana integral. Tres experiencias europeas innovadoras: Île de Nantes, Coin Street y Barrio de la Mina. Madrid: Sepes.

World Bank. (s.f.). Managing the potential undesirable impacts of urban regeneration: Gentrification and loss of social capital. Recuperado de https://urban-regeneration.worldbank.org/node/45 


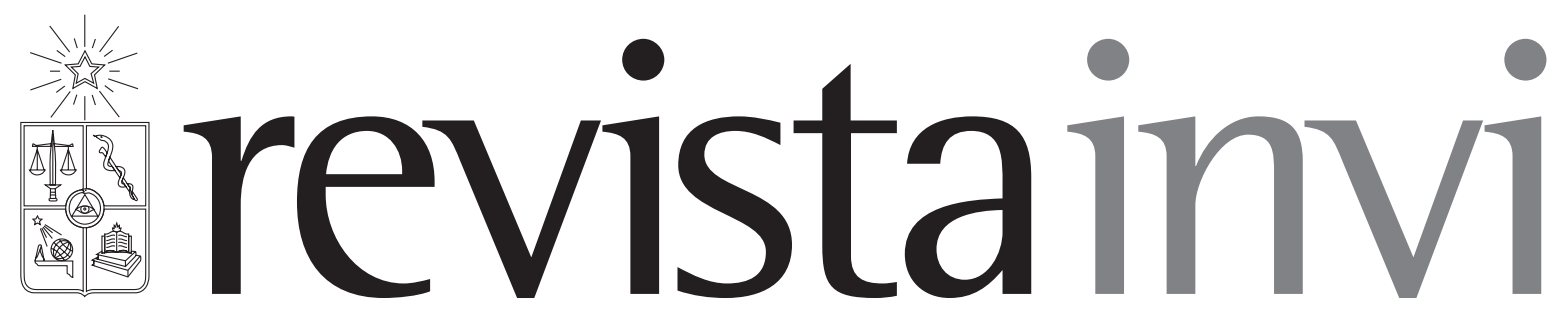

Revista INVI es una publicación periódica, editada por el Instituto de la Vivienda de la Facultad de Arquitectura y Urbanismo de la Universidad de Chile, creada en 1986 con el nombre de Boletín INVI. Es una revista académica con cobertura internacional que difunde los avances en el conocimiento sobre la vivienda, el hábitat residencial, los modos de vida y los estudios territoriales. Revista INVI publica contribuciones originales en español, inglés y portugués, privilegiando aquellas que proponen enfoques inter y multidisciplinares y que son resultado de investigaciones con financiamiento y patrocinio institucional. Se busca, con ello, contribuir al desarrollo del conocimiento científico sobre la vivienda, el hábitat y el territorio y aportar al debate público con publicaciones del más alto nivel académico.

Director: Dr. Ricardo Tapia Zarricueta, Universidad de Chile, Chile.

Editor: Dr. Luis Campos Medina, Universidad de Chile, Chile.

Editor asistente: Dr. Walter Imilan, Universidad de Chile, Chile.

Coeditora: Srta. Sandra Rivera, Universidad de Chile, Chile.

\section{COMITÉ EDITORIAL:}

Dr. Victor Delgadillo, Universidad Autónoma de la Ciudad de México, México.

Dra. María Mercedes Di Virgilio, CONICET/ IIGG, Universidad de Buenos Aires, Argentina.

Dra. Irene Molina, Uppsala Universitet, Suecia.

Dr. Gonzalo Lautaro Ojeda Ledesma, Universidad de Valparaíso, Chile.

Dra. Suzana Pasternak, Universidade de São Paulo, Brasil.

Dr. Javier Ruiz Sánchez, Universidad Politécnica de Madrid, España.

Dra. Elke Schlack Fuhrmann, Pontificia Universidad Católica de Chile, Chile.

Dr. Carlos Alberto Torres Tovar, Universidad Nacional de Colombia, Colombia.

Sitio web: http://www.revistainvi.uchile.cl/

Correo electrónico: revistainvi@uchilefau.cl

Licencia de este artículo: Creative Commons Atribución-Compartirlgual 4.0 Internacional (CC BY-SA 4.0) 